\title{
Results on the Spin Structure of the Nucleon from COMPASS
}

\author{
F. Kunne ${ }^{\mathrm{a}}$, on behalf of the COMPASS Collaboration \\ ${ }^{a}$ CEA Saclay IRFU/SPhN, 91191 Gif/Yvette, France
}

\begin{abstract}
Highlights of the nucleon spin studies from the COMPASS experiment at CERN are presented. Three independent measurements of the gluon polarization give values compatible with zero for $x$ close to 0.1 . The quark helicity distributions are determined for all flavors. On the transversity side, Collins and Sivers asymmetries are measured for proton and deuteron targets. Plans for COMPASS-II include DVCS as well as polarized Drell-Yan measurements.
\end{abstract}

Keywords: Spin of the Nucleon, gluon and quark helicity, transversity, polarization PACS: 13.88.+e

\section{INTRODUCTION}

The main objective of the COMPASS muon physics program is the study of the nucleon spin. COMPASS has accumulated data during 5 years using the CERN SPS $160 \mathrm{GeV}$ polarized muon beam and a longitudinally or transversely polarized deuteron $\left({ }^{6} \mathrm{LiD}\right)$ or proton $\left(\mathrm{NH}_{3}\right)$ target. We present here results obtained on the gluon and quark helicities and, for the transverse sector, Collins and Sivers asymmetries. Finally, we briefly present plans for the future COMPASS-II experiment.

\section{LONGITUDINAL SPIN}

The nucleon spin can be decomposed into contributions from quarks, gluons and orbital angular momentum as $1 / 2=1 / 2 \Delta \Sigma+\Delta G+L_{z}$. Predictions from the naive quark parton model as well as from a QCD approach neglecting the strange quark polarization lead to a large polarization of the quarks, i.e. $\Delta \Sigma=0.6$. On the contrary, measurements from the last decades show that $\mathrm{a}_{0}$, the singlet axial matrix element related to $\Delta \Sigma$, is small, $\sim 0.3$. In fact, in some QCD schemes, $\mathrm{a}_{0}=\Delta \Sigma-\left(3 \alpha_{s} / 2 \pi\right) \Delta \mathrm{G}$. Thus a large value of $\Delta \mathrm{G}$ could help restoring $\Delta \Sigma \sim 0.6$. This fact, together with the $1 / 2$ nucleon spin sum rule, has motivated the direct measurements of $\Delta \mathrm{G}$. This can be performed via the double spin asymmetry of cross sections for the photon gluon fusion (PGF) process. PGF events are searched for in two channels: the 'open charm' channel where an outgoing charm quark is identified via the production of $\mathrm{D}^{\circ}$ mesons, and the 'high $\mathrm{p}_{\mathrm{T}}$ hadron pair' channel, where the two outgoing quarks (likely light quarks) hadronize with high transverse momentum. The open charm channel, only accessible at COMPASS, provides a clean signature of the PGF but with limited statistics. On the contrary, for the high $\mathrm{p}_{\mathrm{T}}$ channel, the statistics is high but there are competing

CP1317, QCD@WORK 2010, International Workshop on Quantum Chromodynamics: Theory and Experiment edited by L. Angelini, G. E. Bruno, P. Colangelo, D. Creanza, F. De Fazio, and E. Nappi

(C) 2010 American Institute of Physics 978-0-7354-0872-2/10/\$30.00 
background processes which have to be simulated. Figure 1 shows all existing direct

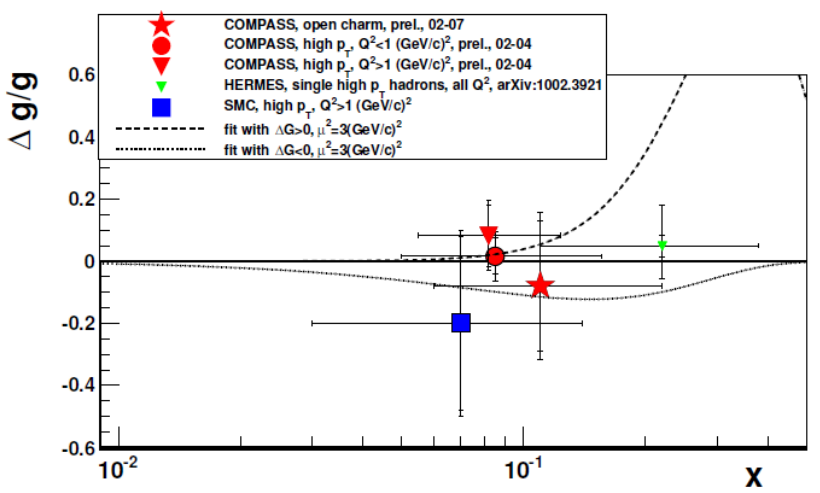

FIGURE 1. Gluon polarization $\Delta \mathrm{G} / \mathrm{G}(\mathrm{x})$ : COMPASS results from the open charm (star) and high $\mathrm{p}_{\mathrm{T}}$ hadron (large triangle and circle) channels are shown together with HERMES (small triangle) and SMC (square) results and with two QCD fits to $g_{1}$.

measurements of the gluon polarization $\Delta G / G(x)$. COMPASS results [1] from the open charm (star) and high $\mathrm{p}_{\mathrm{T}}$ hadron (large triangle and circle) channels are shown together with HERMES (small triangle) and SMC (square) results. The results probe $x$ values around $\sim 0.1-0.2$, and are all compatible with zero in this kinematic range. The curves describe the two solutions, one positive and one negative, from the COMPASS QCD fits to the $g_{1}$ world data. For both fits, the resulting first moment of $\Delta G$ is of the order of 0.2-0.3 in absolute value. From all the present experimental results, including RHIC spin data, one concludes on a first moment of $\Delta G$ smaller than $\sim 0.35$. The consequence for the possible decomposition of the nucleon spin on its constituents is summarized in the Table 1 , where three scenarios with minimal, zero and maximal $\Delta \mathrm{G}$ at a scale of $3(\mathrm{GeV} / \mathrm{c})^{2}$ are shown.

TABLE 1. Three possible scenarios for the nucleon spin decomposition

\begin{tabular}{lccc}
\hline & $1 / 2 \Delta \Sigma$ & $\Delta \mathbf{G}$ & $\mathbf{L z}$ \\
\hline Scen.1, $\Delta \mathrm{G}$ max $<0$ & 0.15 & 0.35 & 0. \\
Scen.2 $\Delta \mathrm{G}=0$ & 0.15 & 0. & 0.35 \\
Scen.3, $\Delta \mathrm{G}$ max $>0$ & 0.15 & -0.35 & 0.70 \\
\hline
\end{tabular}

In parallel to the $\Delta \mathrm{G}$ measurement, data on deep inelastic scattering $\mu \mathrm{p} \rightarrow \mu \mathrm{X}$ (DIS) are recorded. They are used to extract the spin structure functions, $g_{1}{ }^{p}$ and $g_{1}{ }^{d}$, of the proton [2] and deuteron (Fig.2). Global QCD fits at NLO of world data on $g_{1}$ are used to extract the quark and gluon helicity distributions $\Delta \mathrm{q}\left(x, Q^{2}\right)$ and $\Delta \mathrm{G}\left(x, Q^{2}\right)$. Semi inclusive DIS (SIDIS) events where an additional hadron tags the flavor of the struck quark are used to extract at LO helicity quark distributions for all flavors. This provides a wider picture of the nucleon spin, however requiring an additional input, the quark fragmentation functions (FF). COMPASS results [3] obtained using DSS FFs are shown in Fig.3 left, together with HERMES ones and a global LO QCD fit. In 
Fig.3 right, the strange quark helicity is compared to a more recent NLO global fit
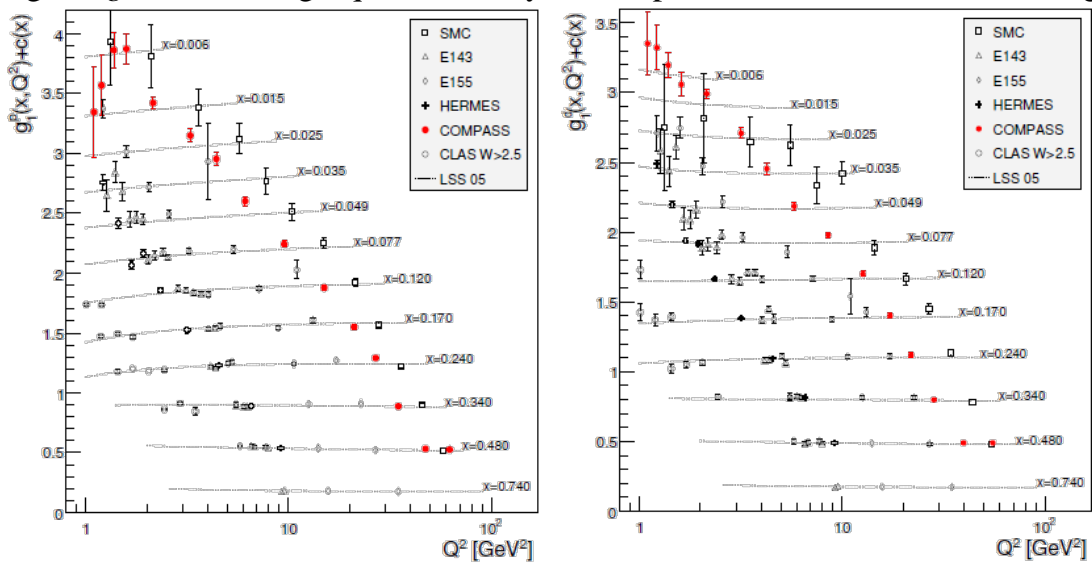

FIGURE 2. Spin structure function of the proton (left) and deuteron (right) $v s \mathrm{Q}^{2}$, for various $x$.

from DSSV which accommodates both for the COMPASS and HERMES SIDIS data which are compatible with zero, and with the results from analyses of inclusive DIS data, which lead to a negative first moment for $\Delta \mathrm{s}$.

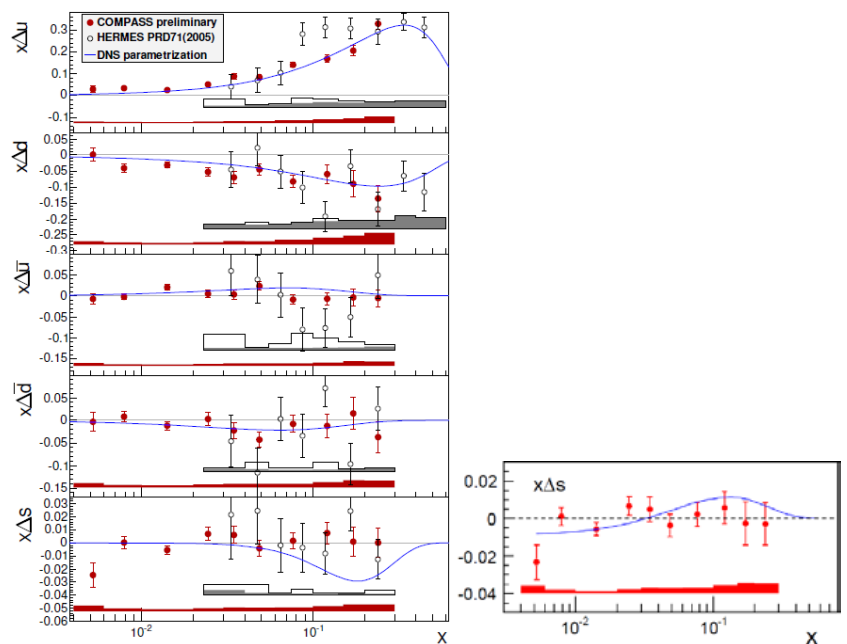

FIGURE 3. Left: COMPASS (closed points) and HERMES (open points) results for the helicity quark distribution $\mathrm{x} \Delta \mathrm{q}(\mathrm{x})$ for five flavors compared to the LO global QCD fit of DNS. Right: The strange quark helicity distribution $\mathrm{x} \Delta \mathrm{s}(\mathrm{x})$ compared to the more recent NLO global QCD fit of DSSV.

\section{TRANSVERSE SPIN}

Three structure functions are necessary to describe the nucleon at leading twist: $\mathrm{F}_{1}(x), \mathrm{g}_{1}(x)$ and $\mathrm{h}_{1}(x)$. The latter is linked to the distribution of transversely polarized 
partons. It can be accessed in SIDIS with a transversely polarized target, when coupled to an adequate FF. The azimuthal asymmetry of hadrons, the 'Collins' asymmetry, is sensitive to $h_{1}(x)$. COMPASS has published the asymmetries measured with a deuteron target, which are compatible with zero. In Fig.4 top, the results [4] obtained with a transversely polarized proton target are shown for positively and negatively charged hadrons. The data extend towards ten times lower $x$ than HERMES. They are compared to a prediction [5] based on a combined analysis of COMPASS deuteron, HERMES proton and BELLE FF data. The analysis concludes that $\Delta_{\mathrm{T}} \mathrm{u}(x)$ is positive and $\Delta_{\mathrm{T}} \mathrm{d}(x)$ negative, and both smaller than helicity distributions.
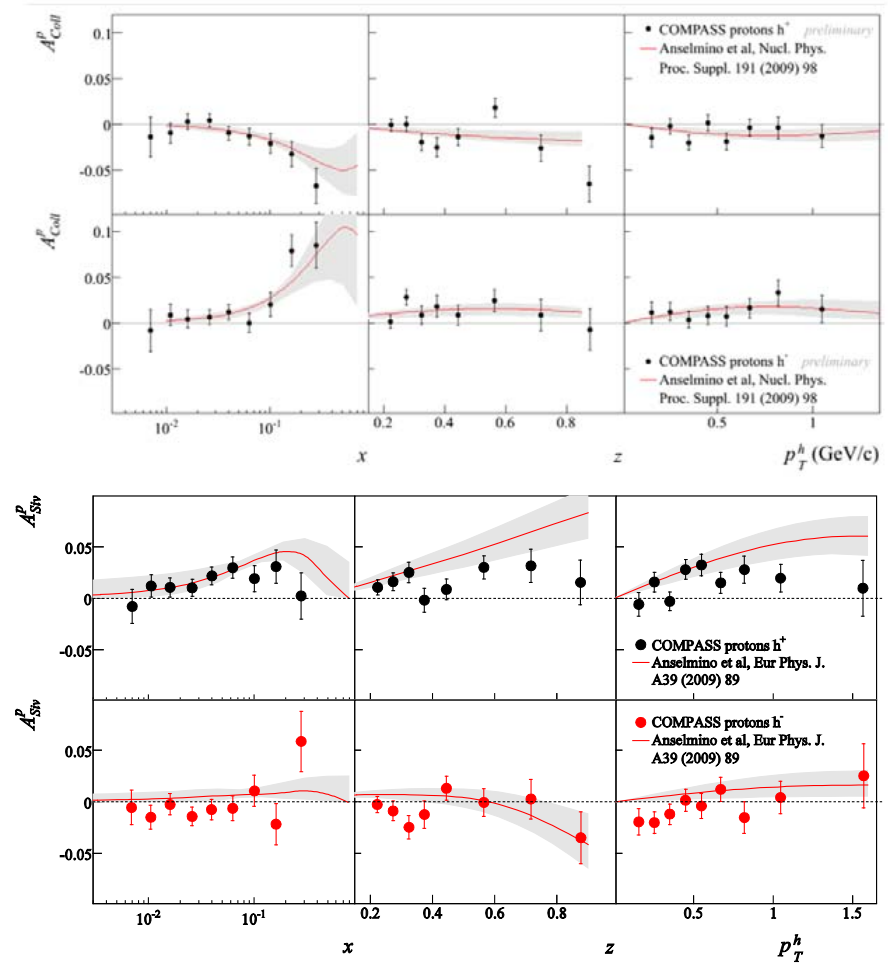

FIGURE 4. Collins (top plot) and Sivers (bottom plot) asymmetries on proton $v s \quad x, z$ and $p_{T}$, for positive (up) and negative (down) hadrons. The curve is a global QCD analysis of Anselmino et al.[5].

Simultaneously, another independent azimuthal asymmetry is measured, the 'Sivers' asymmetry, which is sensitive to the correlation between the nucleon spin and the transverse momentum of quarks. COMPASS results on the deuteron are compatible with zero, while the results on the proton, shown in Fig.4 bottom, present a slight signal at high $x$ for positively charged hadrons. The present signal is smaller than the one seen by HERMES, however in a slightly different kinematical range. Combined analyses of COMPASS deuteron and HERMES proton data provide the Sivers functions for the various quark flavours [5]. Other azimuthal asymmetries, not 
shown here, are measured simultaneously. When analyzed in global fits, they should impose constraints on the transverse momentum dependent (TMD) distributions.

\section{COMPASS-II}

In the future COMPAS-II experimental programme, foreseen for 2012 onwards, two new sectors will be studied: the transverse imaging of the nucleon via exclusive processes, and the TMD distributions via polarized Drell-Yan reactions.

\section{Transverse imaging via exclusive processes: DVCS and DVMP}

Generalized parton distributions (GPDs) provide a unified description of form factors and parton distribution functions, and a transverse imaging of the nucleon. Several observables sensitive to GPDs will be measured. They are accessible through exclusives processes like the deep virtual Compton scattering ( $\mu \mathrm{p} \rightarrow \mu \mathrm{p} \gamma$ DVCS) or the deep virtual meson production (DVMP). Fig.5 left shows an example of projected error bars for a two year measurement of the 'Beam Charge and Spin' asymmetry obtained in the interference of the DVCS and the Bethe-Heitler $(\mathrm{BH})$ processes. The same quantity will be measured in various other kinematical bins, covering an $x$ range between 0.01 and 0.1, i.e. intermediate between the DESY and JLab ranges. Fig.5 right shows results from a short test run from 2009, done to study the feasibility of the experiment. Events with exclusive production of photons are compared to a simulation in 3 different $x$ bins. While at lower $x$ (first histogram) the $\mathrm{BH}$ events are expected to dominate, at high $x$ (third histogram), the $\mathrm{BH}$ fraction is expected to be small (dotted curve) and the total signal of DVCS+BH (full curve) is high. The data in the third histogram thus sign the presence of DVCS events, as expected at high $x$.

In parallel to the DVCS and DVMP measurements, high statistics on SIDIS reactions

$\mu \mathrm{p} \rightarrow \mu \mathrm{ph}$ will be recorded. Azimuthal hadron asymmetries will give access to several TMDs. In addition, a full mapping of pion and kaon multiplicities will serve as input to global NLO QCD analyses of quark FF on one side, and of PDFs on the other one. A LO approach can also be used to extract for instance the strange distribution function $s(x)$. Projected errors for one week of data using DSS FF are shown in Fig.6 together with HERMES data covering a reduced $x$ range and with MSTW and CTEQ parameterizations. 

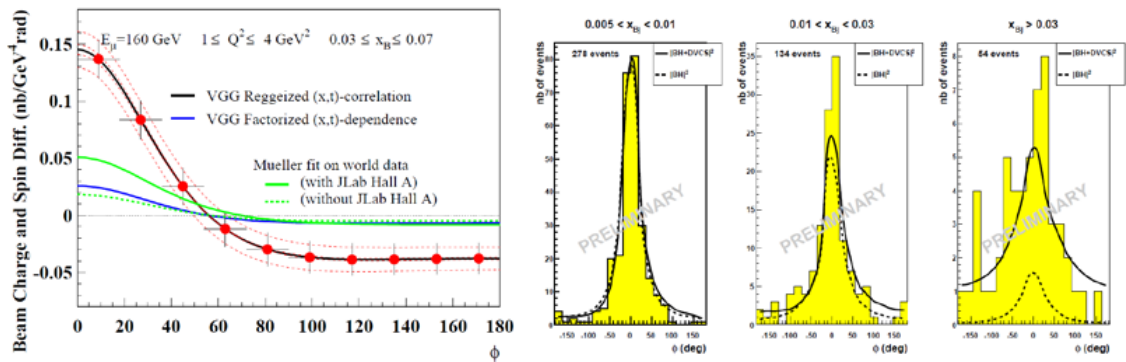

FIGURE 5. Left: COMPASS-II projection for an asymmetry sensitive to $x$ and $t$ correlations. Right: results from a short test run showing events with exclusive production of photons in 3 different $\mathrm{x}$ bins. The full curve corresponds to DVCS+BH, while the dotted curve shows BH only.

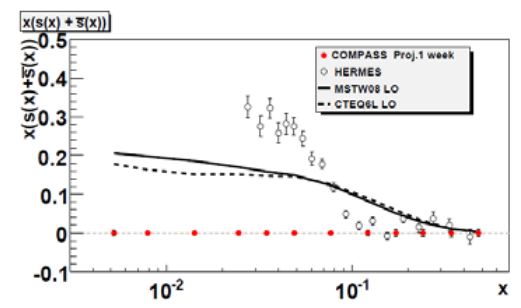

FIGURE 6. COMPASS-II projection error bars for $x \Delta \mathrm{s}(x)$.

\section{TMD distributions via polarized Drell-Yan}

Using the high energy pion beam in conjunction with the transversely polarized proton target $\left(\mathrm{NH}_{3}\right)$ and detecting Drell-Yan (DY) events via muon pairs, COMPASSII will be able to measure asymmetries sensitive to various TMDs, like the Sivers and Boer-Mulders ones. These data will provide a test of the factorization approach: the sign of the Sivers function is expected to be opposite in the DY and SIDIS reactions and this has never been tested experimentally yet. A first short test run was realized in 2009 to study the feasibility of such a measurement, and the resulting invariant mass spectrum of the dimuon pair is shown in Fig.7 left. Projected error bars for the COMPASS-II two year measurement of an azimuthal asymmetry sensitive to the Sivers function is shown in Fig.7 right. The curves show how the various theoretical predictions differ.
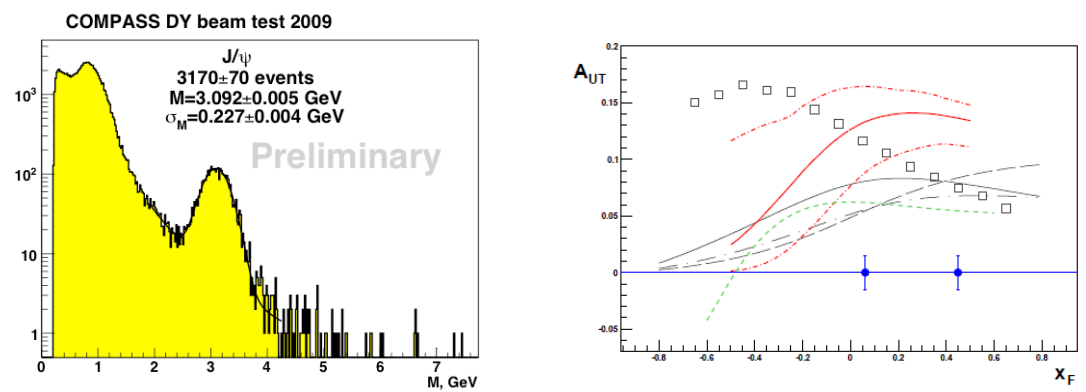

FIGURE 7. Left: Data sample of DY events from a short test run. Right: Error bar projection for 2 years for the azimuthal asymmetry in the polarized DY process, and various theoretical predictions 


\section{REFERENCES}

1. Franco, Proceedings of the DIS-2010 conference; COMPASS Coll. Phys.Lett.B 63325 (2006).

2. COMPASS Coll. Phys.Lett.B 690466 (2010),

3. COMPASS Coll., hep-ex/1007.4061, submitted to \{lit Phys. Lett.\} B.

4. COMPASS Coll., hep-ex/1005.5609, sub. Phys.Lett.B

5. M.Anselmino et al, arXiv:0812.4366; M.Murgia, contribution to this conference. 